\title{
Hybrid Functional Interpretations
}

\author{
Mircea-Dan Hernest ${ }^{1}$ and Paulo Oliva ${ }^{2, \star}$ \\ ${ }^{1}$ Informatics Institute, University of Innsbruck, Austria \\ dan.hernest@uibk.ac.at \\ ${ }^{2}$ Department of Computer Science, Queen Mary, University of London \\ pbo@dcs . qmul . ac . uk
}

\begin{abstract}
We show how different functional interpretations can be combined via a multi-modal linear logic. A concrete hybrid of Kreisel's modified realizability and Gödel's Dialectica is presented, and several small applications are given. We also discuss how the hybrid interpretation relates to variants of Dialectica and modified realizability with non-computational quantifiers.
\end{abstract}

Keywords: Functional interpretations, modified realizability, Dialectica interpretation, linear logic, program extraction from proofs, uniform quantifiers.

\section{Introduction}

The second author recently devised a unified presentation [1] of different functional interpretations of intuitionistic logic, including Kreisel's modified realizability [2] and Gödel's Dialectica interpretation [3]. As it turns out, these distinct interpretations diverge only in the treatment of the structural rule of contraction. Therefore, due to its finer handling of contractions, linear logic [4] gives us the optimal setting for further analysing and comparing these interpretations [5,6].

In this article we show that functional interpretations not only can be better understood modulo linear logic, but can also be successfully combined into what we term hybrid interpretations, where features of different interpretations can coexist. Consider for instance the handling of extensionality when working in the language of all finite types. In the case of modified realizability we can safely adopt a fully extensional setting with primitive equality for basic types (say $n=m$ for numbers $n, m \in \mathbb{N}$ ) and higher-type equality defined as $f \stackrel{\rho \rightarrow \tau}{=} g: \equiv \forall x^{\rho}(f x \stackrel{\tau}{=} g x)$ together with the axiom schema of extensionality

$$
x \stackrel{\rho}{=} y \rightarrow f x \stackrel{\tau}{=} f y
$$

for all finite types $\rho, \tau$. However, when it comes to Dialectica interpretation, the translation of (1) requires witnesses for the universal quantifiers within $x \stackrel{\rho}{=} y$, which cannot be majorised in general [7] and hence cannot be expressed inside Gödel's system T. But recall that intuitionistic proofs can be embedded into linear logic ones, with intuitionistic implications $A \rightarrow B$ translated as linear implications $! A \multimap B$. The difficulty

\footnotetext{
* The second author gratefully acknowledges support of the Royal Society of the UK under grant 516002.K501/RH/kk.
}

A. Beckmann, C. Dimitracopoulos, and B. Löwe (Eds.): CiE 2008, LNCS 5028, pp. 251-260 2008.

(C) Springer-Verlag Berlin Heidelberg 2008 
of Dialectica in dealing with full extensionality is that the "negative information" in the assumption $! A \equiv !(x \stackrel{\rho}{=} y)$ of (1) should not (and cannot) be witnessed. In other words, the modality "!" should in this case rather be treated as by Kreisel's modified realizability, also when carrying out a Dialectica interpretation.

This distinguished treatment of the modalities is possible because, as pointed out by Girard (cf. [8] and [9], p84), the modalities are not canonical, thus different modalities can coexist into a single system. Therefore, we intend to make use of what we name a multi-modal linear logic, which includes distinct modalities corresponding to each of the various functional interpretations. E.g., in the extensionality example (1) we rather use Kreisel's modified realizability modality $\left(!_{k} A\right)$ in order to express that the information in the premise of the axiom schema should not be witnessed:

$$
!_{k}(x \stackrel{\rho}{=} y) \multimap f x \stackrel{\tau}{=} f y .
$$

This generalises Spector's quantifier-free rule of extensionality (see [10]) since it allows us to derive $r s \stackrel{\tau}{=} r t$ from $s \stackrel{\rho}{=} t$ in any context of the form $!_{k} \Delta$, and has the advantage that visibly (2) requires no realizer.

In contrast to modified realizability, the Dialectica interpretation is well-suited to deal with classical proofs via negative translation, as it interprets the Markov principle

$$
\neg \forall x A_{\mathrm{qf}}(x) \rightarrow \exists x \neg A_{\mathrm{qf}}(x) .
$$

Since the premise of (3) corresponds in linear logic to ? $\exists x A_{\mathrm{qf}}^{\perp}(x)$, the modality "?" should rather be treated as by the Dialectica interpretation, even when attempting to do a modified realizability, hence (3) should be replaced by

$$
!_{g} ?_{g} \exists x A_{\mathrm{qf}}(x) \multimap \exists x ?_{g} A_{\mathrm{qf}}(x) .
$$

For proofs which use both extensionality (2) and Markov principle (4), constructive information will be extracted whenever such a labelling of the modalities is possible.

The setting of multi-modal linear logic also allows for a unified study of the noncomputational ("nc" for short) quantifiers introduced by Berger in the context of modified realizability [11] and adapted by the first author to Dialectica interpretation [12].

The paper is organised as follows. In the next section we present the formal system of multi-modal linear logic. In Section 3 we introduce the hybrid functional interpretation of the multi-modal system. In Section 4 we present a few illustrative applications of the hybrid interpretation. A comparison between the use of nc quantifiers and of our hybrid logic is given in Section 5. Section 6 discusses possible extensions of the hybrid interpretation to include other modalities. In Section 6.1 we present some ideas for an algorithm which decorates a (linear translation of a) given intuitionistic proof with different modalities so as to achieve a desired outcome of the extraction program.

\section{Multi-modal Linear Logic $L_{h}^{\omega}$}

We build upon an extension of classical linear logic to the language of all finite types, introduced by the second author in [13]. The set of finite types $\mathcal{T}$ is inductively defined 
by: $i, b \in \mathcal{T}$ and if $\rho, \sigma \in \mathcal{T}$ then $\rho \rightarrow \sigma \in \mathcal{T}$. For simplicity, we deal with only two basic finite types $i$ (integers) and $b$ (booleans). We use no linear types nor linear terms.

We assume that the terms of $\operatorname{LL}_{h}^{\omega}$ contain all typed $\lambda$-terms, i.e. variables $x^{\rho}$ for each finite type $\rho, \lambda$-abstractions $\left(\lambda x^{\rho} \cdot t^{\sigma}\right)^{\rho \rightarrow \sigma}$, term applications $\left(t^{\rho \rightarrow \sigma} s^{\rho}\right)^{\sigma}$, and conditionals $\left(s^{b}\right)\left(t^{\rho}, r^{\rho}\right)$. The atomic formulas of $\mathrm{LL}_{\mathrm{h}}^{\omega}$ are $A_{\text {at }}, B_{\text {at }}, \ldots$ and $A_{\text {at }}^{\perp}, B_{\text {at }}^{\perp}, \ldots$. For simplicity, the standard propositional constants $0,1, \perp, \top$ of linear logic have been omitted, since the hybrid interpretation of atomic formulas is trivial (see Definition 1).

Formulas are built from atomic formulas via connectives $A \ngtr B$ (par), $A \otimes B$ (tensor), $A \diamond_{z} B$ (if-then-else) and quantifiers $\forall x A$ and $\exists x A$. Exponentials will be treated in Section 2.1 and nc-quantifiers in Section 5 . The linear implication $A \multimap B$ abbreviates $A^{\perp} 8 B$ where the linear negation $(\cdot)^{\perp}$ is an abbreviation so that $\left(A^{\perp}\right)^{\perp}$ is syntactically equal to $A$ (see [4, 13]). Recall that the structural rules of linear logic do not include the usual rules of weakening and contraction. These are added separately, in a controlled manner via the use of modalities (cf. Section 2.1). The rules for the multiplicative connectives and quantifiers are the usual ones for (one-sided) classical linear logic (see [4] 13]). Following [5], we deviate from the standard formulation of linear logic and use the if-then-else logical constructor $A \diamond_{z} B$ instead of standard additive conjunction and disjunction]. The rules for $A \diamond_{z} B$ are given in [13] (Table 3). In terms of quantification over booleans, the standard additives can be defined as

$$
A \wedge B: \equiv \forall z^{b}\left(A \diamond_{z} B\right) \quad A \vee B: \equiv \exists z^{b}\left(A \diamond_{z} B\right)
$$

Notation for tuples. We use bold face variables $\boldsymbol{f}, \boldsymbol{g}, \ldots, \boldsymbol{x}, \boldsymbol{y}, \ldots$ for tuples of variables, and bold face terms $\boldsymbol{a}, \boldsymbol{b}, \ldots, \boldsymbol{\gamma}, \boldsymbol{\delta}, \ldots$ for tuples of terms. Given the sequences of terms $\boldsymbol{a}$ and $\boldsymbol{b}$, by $\boldsymbol{a}(\boldsymbol{b})$ we mean the sequence of terms $a_{0}(\boldsymbol{b}), \ldots, a_{n}(\boldsymbol{b})$. Similarly for the multiple simultaneous substitution $\boldsymbol{a}[\boldsymbol{b} / \boldsymbol{x}]$.

\subsection{Kreisel and Gödel Modalities}

The second author [5, 13] has recently studied possible different interpretations for the exponentials! and ?, and how these correspond to well-known functional interpretations of intuitionistic logic. We here introduce syntactically distinct exponentials (see Table 11 and show how these different interpretations can coexist (whence the "hybrid" denomination). For simplicity we have considered only the so-called "Kreisel" and "Gödel" modalities, denoted $!_{k}$ and respectively $!_{g}$, together with their duals $?_{k}$

Table 1. Rules for the exponentials $* \in\{k, g\}$

$$
\frac{?_{*} \Gamma, A}{?_{*} \Gamma, !_{*} A}\left(!_{*}\right) \quad \frac{\Gamma, A}{\Gamma, ?_{*} A}\left(?_{*}\right) \quad \frac{\Gamma, ?_{*} A, ?_{*} A}{\Gamma, ?_{*} A}\left(\mathrm{con}_{*}\right) \quad \frac{\Gamma}{\Gamma, ?_{*} A}\left(\mathrm{wkn}_{*}\right)
$$

\footnotetext{
${ }^{1}$ See Girard's comments in [4] (p13) and [9] (p73) on the relation between the additive connectives and the if-then-else construct.
} 
and $?_{g}$. This will correspond to a combination of modified realizability and Dialectica interpretation into a single functional interpretation which supersedes both of them.

Moreover, a partial order of information is put on the distinct modalities in the form of the following "relaxing" rules

$$
\frac{\Gamma, ?_{g} A}{\Gamma, ?_{k} A}(\text { ?-relax }) \quad \text { and } \quad \frac{\Gamma, !_{k} A}{\Gamma, !_{g} A}(! \text {-relax })
$$

meaning that at anytime we can choose to "forget" some information we had. This is because, as will be reflected in the hybrid interpretation given below, the Gödel "whynot" is meant to carry a finer information than $?_{k}$, whereas the Kreisel "bang" is more general than $!_{g}$. The usual rules for both kinds of exponentials are presented in Table 1 .

In mixing both Kreisel's and Gödel's interpretations, we must add also the following restriction on the "Gödel" contraction rule $\operatorname{con}_{g}$ (for terminology see Section 3 below):

(*) if the contraction formula $A$ in $\operatorname{con}_{g}$ is computationally relevant, then it must not contain any Kreisel whynot $?_{k}$ in front of a computationally relevant subformula, and also no Kreisel bang $!_{k}$ in front of a refutation relevant subformula.

As we will see, $(*)$ ensures that the interpretation of such formulas $A$ is quantifier-free (hence decidable); $(*)$ is necessary and sufficient for attaining Theorem 1

\section{A Hybrid Functional Interpretation}

To each formula $A$ of $L L_{\mathrm{h}}^{\omega}$ we associate a not necessarily quantifier-free formula $|A|_{\boldsymbol{y}}^{\boldsymbol{x}}$ of linear logic $L^{\omega}{ }^{\omega}$ (defined in [13]) where $\boldsymbol{x}, \boldsymbol{y}$ are fresh variables not appearing in $A$. The length and types of $\boldsymbol{x}, \boldsymbol{y}$ are inductively determined by the formula $A$. The variables $\boldsymbol{x}$ in the superscript are called the witnessing variables, while the subscript variables $\boldsymbol{y}$ are called the challenge variables. Intuitively, the interpretation of $A$ is a two-player (Eloise and Abelard) one-move game, where $|A|_{\boldsymbol{y}}^{\boldsymbol{x}}$ is the adjudication relation. We want that Eloise has a winning move whenever $A$ is provable in $L_{h}^{\omega}$. Moreover, the hybrid linear logic proof of $A$ will provide Eloise's winning move $\boldsymbol{a}$, i.e., $\forall \boldsymbol{y}|A|_{\boldsymbol{y}}^{\boldsymbol{a}}$ will hold in $\mathrm{LL}^{\omega}$, where $\boldsymbol{a}$ is a tuple of terms of corresponding types.

Formulas for which the tuple of witnessing variables is not empty are considered computationally relevant, and formulas for which the sequence of challenge variables is not empty are considered refutation relevant. An $?_{k}$ (respectively $!_{k}$ ) in front of a computationally (respectively refutation) irrelevant formula will be called redundant.

Definition 1 (Hybrid Interpretation). The interpretation of atomic formulas are the atomic formulas themselves, with empty sets of witnessing and challenge variables, i.e. $\left|A_{\mathrm{at}}\right|: \equiv A_{\mathrm{at}}$ and $\left|A_{\mathrm{at}}^{\perp}\right|: \equiv A_{\mathrm{at}}^{\perp}$. Assuming $|A|_{\boldsymbol{y}}^{\boldsymbol{x}}$ and $|B|_{\boldsymbol{w}}^{\boldsymbol{v}}$ already defined, we define

$$
\begin{aligned}
|A \diamond B|_{\boldsymbol{y}, \boldsymbol{w}}^{\boldsymbol{f}, \boldsymbol{g}} & : \equiv|A|_{\boldsymbol{y}}^{\boldsymbol{f} \boldsymbol{w}} \diamond|B|_{\boldsymbol{w}}^{\boldsymbol{g y}} & |\exists z A(z)|_{\boldsymbol{f}}^{\boldsymbol{x}, z}: \equiv|A(z)|_{\boldsymbol{f} z}^{\boldsymbol{x}} \\
|A \otimes B|_{\boldsymbol{f}, \boldsymbol{g}}^{\boldsymbol{x}, \boldsymbol{v}} & : \equiv|A|_{\boldsymbol{f} \boldsymbol{v}}^{\boldsymbol{x}} \otimes|B|_{\boldsymbol{g} \boldsymbol{x}}^{\boldsymbol{v}} & |\forall z A(z)|_{\boldsymbol{y}, z}^{\boldsymbol{f}}: \equiv|A(z)|_{\boldsymbol{y}}^{\boldsymbol{f} z} \\
\left|A \diamond_{z} B\right|_{\boldsymbol{y}, \boldsymbol{w}}^{\boldsymbol{x}, \boldsymbol{v}} & : \equiv|A|_{\boldsymbol{y}}^{\boldsymbol{x}} \diamond_{z}|B|_{\boldsymbol{w}}^{\boldsymbol{v}} . &
\end{aligned}
$$


Finally, we can give different interpretations to the modalities as:

$$
\begin{array}{rlrl}
\left|!_{k} A\right|^{\boldsymbol{x}} & : \equiv ! \forall \boldsymbol{y}|A|_{\boldsymbol{y}}^{\boldsymbol{x}} & \left|!_{g} A\right|_{\boldsymbol{f}}^{\boldsymbol{x}}: \equiv !|A|_{\boldsymbol{f} \boldsymbol{x}}^{\boldsymbol{x}} \\
\left|?_{k} A\right|_{\boldsymbol{y}}: \equiv ? \exists \boldsymbol{x}|A|_{\boldsymbol{y}}^{\boldsymbol{x}} & \left|?_{g} A\right|_{\boldsymbol{y}}^{\boldsymbol{f}}: \equiv ?|A|_{\boldsymbol{y}}^{\boldsymbol{f y}} .
\end{array}
$$

It is easy to see that $\left|A^{\perp}\right|_{\boldsymbol{x}}^{\boldsymbol{y}} \equiv\left(|A|_{\boldsymbol{y}}^{\boldsymbol{x}}\right)^{\perp}$ and thus $|A \multimap B|_{\boldsymbol{x}, \boldsymbol{w}}^{\boldsymbol{f}, \boldsymbol{g}} \equiv|A|_{\boldsymbol{f} \boldsymbol{w}}^{\boldsymbol{x}} \multimap|B|_{\boldsymbol{w}}^{\boldsymbol{g x}}$.

We prove the soundness of our interpretation, i.e., we show how Eloise's winning move in the game $|A|_{\boldsymbol{y}}^{\boldsymbol{x}}$ can be algorithmically extracted from a proof of $A$ in $\mathrm{LL}_{\mathrm{h}}^{\omega}$.

Theorem 1 (Soundness of Hybrid Interpretation). Let $A_{0}, \ldots, A_{n}$ be a sequence of formulas of $\mathrm{LL}_{\mathrm{h}}^{\omega}$, with $z$ as the only free-variables. If the sequent $A_{0}, \ldots, A_{n}$ is provable in $\mathrm{LL}_{\mathrm{h}}^{\omega}$, then terms $\boldsymbol{a}_{0}, \ldots, \boldsymbol{a}_{n}$ can be automatically synthesised from its formal proof, such that the translated sequent $\left|A_{0}\right|_{\boldsymbol{x}_{0}}^{\boldsymbol{a}_{0}}, \ldots,\left.\left|A_{n}\right|_{\boldsymbol{x}_{n}}\right|_{\boldsymbol{x}_{n}}$ is provable in $\mathrm{LL}^{\omega}$, where $\mathrm{FV}\left(\boldsymbol{a}_{i}\right) \in\left\{\boldsymbol{z}, \boldsymbol{x}_{0}, \ldots, \boldsymbol{x}_{n}\right\} \backslash\left\{\boldsymbol{x}_{i}\right\}$.

Proof: Ignoring the rules for Gödel exponentials, the proof is given in [5], with $!: \equiv !_{k}$ and $?: \equiv ?_{k}$. The addition of Gödel exponentials to the language (together with their interpretation) does not alter the facts. Also the rules for Gödel exponentials are treated in [13], but independent of the Kreisel exponentials. Hence all we need to prove is that the proofs in [13] still hold after adding Kreisel exponentials to the language (together with their interpretation). It is easy to notice that, due to the restriction $(*)$ we added on $\operatorname{con}_{g}$, the interpretation of $?_{g} A$ is quantifier-free, hence decidable. This is because (so far) only non-redundant $!_{k}$ or $?_{k}$ could introduce quantifiers in the translated formula. $\square$

\section{Simple Applications to Program Extraction}

In this section we present some examples where it pays off to analyse proofs using both Kreisel and Gödel modalities. Some information might not be relevant while some other might be. One can thus use $!_{k} A$ and $?_{k} A$ to ignore the computationally irrelevant parts of the proof, in a way very similar in effect with light Dialectica [12].

\subsection{Example 1}

Consider theorems of the form

$$
\forall x A \rightarrow \forall y B \rightarrow \forall z C
$$

possibly with parameters, where the negative information on $x$ is irrelevant, while the one on $y$ is of our interest. In this case, we would rather view this theorem as

$$
!_{k} \forall x A \multimap !_{g} \forall y B \multimap \forall z C .
$$

For instance, consider the simple intuitionistic theorem

$$
\forall f(\forall n(f(n) \leq 1) \rightarrow \forall m(f(m) \neq f(m+1)) \rightarrow \forall l(f(l)=f(l+2))) .
$$

From a proof of this, using labelling (6), our hybrid interpretation extracts a realizer $\Phi(f, l)$ s.t.

$$
\forall f, l(\forall n(f(n) \leq 1) \rightarrow(f(\Phi(f, l)) \neq f(\Phi(f, l)+1)) \rightarrow(f(l)=f(l+2))) .
$$

Indeed, one such witness is $\Phi(f, l):=$ if $(f(l+1)=f(l+2))$ then $l+1$ else $l$. 


\subsection{Example 2}

More concretely, we consider the well-known example of extracting the Fibonacci numbers from a minimal logic proof of their weak existence. The example was first used in [14] to illustrate the so-called "refined A-translation" and then in [15] to illustrate the light Dialectica (see also Section 4.3 of [12]). The semi-classical Fibonacci proof is a minimal-logic proof of $\forall n \exists^{\mathrm{cl}} m G(n, m)$, where

$$
\exists^{\mathrm{cl}} m G(n, m) \quad: \equiv \quad \forall m(G(n, m) \rightarrow \perp) \rightarrow \perp
$$

from assumptions expressing that $G$ is the graph of the Fibonacci function ( $G$ is viewed as a predicate constant without computational content), i.e., $G(0,0), G(1,1)$ and

$$
\forall l_{1}, l_{2}, l_{3}\left(G\left(l_{1}, l_{2}\right) \rightarrow G\left(l_{1}+1, l_{3}\right) \rightarrow G\left(l_{1}+2, l_{2}+l_{3}\right)\right) .
$$

Note that such a specification fits into the form (5) (with $C: \equiv \perp$ ). As was noticed by the first author in [15], the negative universally quantified $l_{1}, l_{2}$ and $l_{3}$ do not need to be witnessed in order to extract an algorithm for computing the Fibonacci numbers as a witness for $m$ as function of $n$. The proof in [14] can thus be translated to a hybrid linear logic proof such that, in the pattern of (6), statement (8) becomes

$$
? g \exists m G(n, m)
$$

and (9) becomes (we tacitly removed a number of redundant $!_{g}$ from the front of $G$ 's)

$$
!_{k} \forall l_{1}, l_{2}, l_{3}\left(G\left(l_{1}, l_{2}\right) \multimap G\left(l_{1}+1, l_{3}\right) \multimap G\left(l_{1}+2, l_{2}+l_{3}\right)\right)
$$

and therefore only $m$ is witnessed, by the usual Fibonacci algorithm defined as $F_{n}:=$ $F_{n-1}+F_{n-2}$ and $F_{1}:=1$ and $F_{0}:=0$.

\subsection{Example 3}

The Dialectica interpretation and modified realizability also treat the induction rule 2

$$
\frac{A(0) \quad A(n) \rightarrow A(n+1)}{A(l)}(\text { IND })
$$

in slightly different ways. In both cases, the proofs of $A(0)$ and $A(n) \rightarrow A(n+1)$ provide a realiser $\boldsymbol{t}[l]$ for the witnessing variables of $A(l)$, i.e., $|A|_{\boldsymbol{y}}^{\boldsymbol{t}}$. However, only during the extraction of $\boldsymbol{t}$ via Dialectica interpretation a functional which refutes $A(n)$ when given a refutation for $A(n+1)$ will also be extracted. Such realizer is nonetheless not used in the construction of the desired term $t$. Therefore we rather always treat induction in the way modified realizability does, even when constructing a Dialectica witness. In our multi-modal setting, this can be achieved by formulating induction as

$$
\frac{A(0) \quad !_{k} A(n) \multimap A(n+1)}{A(l)}(\text { IND })
$$

since the Kreisel modality blocks the witnessing of counter-example flows.

\footnotetext{
${ }^{2}$ The induction stated here corresponds to the induction rule with no open assumption in natural deduction systems.
} 


\subsection{Example 4}

Consider the representation of real numbers as Cauchy sequences of rationals with a fixed rate of convergence. A real number being positive carries the extra information of a lower bound on how far from zero the limit of the sequence can be (cf. [10]). In order to avoid going into the representation level, when analysing the proof that a certain real function $f$ is positive at $x$, i.e. $f(x)>\mathbb{R} 0$, it is often useful to view this as $\exists l\left(f(x)>\mathbb{R} 2^{-l}\right)$. Although witnessing $l$ gives us some lower bound on the value of $f(x)$, the formula $f(x)>\mathbb{R} 2^{-l}$ still carries information on how far above $2^{-l}$ the value of $f(x)$ is. This extra information is usually irrelevant in practice and the purely existential matrix can be treated as quantifier-free, given that we can always forget these witnesses later. When automatising program extraction, it thus proves to be useful to make sure that the interpretation will not witness the innermost existential quantifier at all. This can be achieved by viewing the statement $f(x)>\mathbb{R} 0$ as $\exists l ?_{k}\left(f(x)>\mathbb{R} 2^{-l}\right)$.

\section{Comparison to Light Dialectica}

As we noticed above, the effect of applying the hybrid functional interpretation on the semi-classical Fibonacci proof is equivalent to that of light Dialectica. This is not unexpected, since the two are related by a shared feature: the occultation of certain nonrelevant quantifiers. Whereas light Dialectica needs a stronger restriction on the introduction rule for the nc-universal quantifier, a direct correspondence exists between the so-called $\mathrm{ncm}-\mathrm{FC}$ condition of [12] and the present hybrid-interpretation restriction $(*)$ on contraction formulas of $\operatorname{con}_{g}$. Both have the purpose of ensuring that the translated contraction formula is decidable. We can thus see the hybrid interpretation as a simplification of the light Dialectica. On the other hand, there are situations which the latter can handle, whereas the former cannot. The reason is that a $!_{k} A$ discards all challenge terms of $|A|$ and symmetrically a $?_{k} A$ discards all witness terms of $|A|$. In contrast, by means of nc-quantifiers one can exactly "pick" which variables of $A$ do not need to be witnessed or challenged. In this sense, light Dialectica appears to be finer than the hybrid functional interpretation. Nonetheless, optimal is to have both techniques available in a single interpretation, combining their different syntactic natures. One can then easily choose to use either of them, or even both when necessary. For this reason we designed the following "light" hybrid interpretation, which supersedes both the hybrid and the light interpretations (light Dialectica [12] and light modified realizability [11]). Moreover, the nc-quantifiers could be useful in a purely linear context already.

\subsection{The Light Hybrid Interpretation}

To the language of our system we add the symbols $\bar{\forall}$ and $\bar{\exists}$ for non-computational universal and existential quantifiers respectively, usually abbreviated nc-forall, nc-exists. The hybrid interpretation of the nc quantifiers is

$$
|\bar{\exists} z A(z)|_{\boldsymbol{y}}^{\boldsymbol{x}}: \equiv \exists z|A(z)|_{\boldsymbol{y}}^{\boldsymbol{x}} \quad|\bar{\forall} z A(z)|_{\boldsymbol{y}}^{\boldsymbol{x}}: \equiv \forall z|A(z)|_{\boldsymbol{y}}^{\boldsymbol{x}} \quad,
$$

hence the translated formula includes the "regular" quantifiers corresponding to the nc quantifiers - a further reason, besides the interpretation of non-redundant $!_{k}, ?_{k}$, that 
$|B|_{\boldsymbol{w}}^{\boldsymbol{v}}$ is not quantifier-free for general $B$. Therefore, the restriction on the contraction rule $\operatorname{con}_{g}$ is enhanced so that computationally relevant contraction formulas must not contain any nc-quantifier, besides satisfying condition $(*)$. Moreover, corresponding rules must be devised for the introduction of the new $\bar{\forall}$ and $\bar{\exists}$. These are just copies of the rules $(\forall)$ and $(\exists)$ :

$$
\frac{\Gamma, A}{\Gamma, \bar{\forall} z A}(\bar{\forall}) \quad \frac{\Gamma, A[t / z]}{\Gamma, \bar{\exists} z A}(\bar{\exists})
$$

but in the case of $(\bar{\forall})$ with an extension of the restriction that $z$ is not free in $\Gamma$ : further $z$ must not be free in the terms $t$ of the $(\exists)$ instances in the proof of the premise $\Gamma, A$, nor in the computationally relevant contraction formulas of this proof. Notice the contextdependency of the above restriction, which is better expressed as " $z$ must not be free in the witnessing terms of the translations of $\Gamma, A$, after mining the proof of this premise sequent". In fact, the latter form is both necessary and sufficient, whereas the former is largely sufficient but can be optimised to become necessary as well (just as in [12]).

Theorem 2 (Soundness of light hybrid interpretation). Theorem 1 still holds after the addition of nc-quantifiers with their introduction rules and hybrid interpretation.

Proof: Notice that the presence of nc-quantifiers does not modify the set of free variables of the translated formula (since nc-quantified variables are regular-quantified in the translation). The interpretation of $(\bar{\exists})$ is just an instance of $(\exists)$. Similarly, the interpretation of $(\bar{\forall})$ is an instance of $(\forall)$, but we must check the restriction that $z$ is not free in the witnessed translation $|\Gamma|_{\boldsymbol{v}}^{\gamma}$ of $\Gamma$. The extra restrictions we set on $(\bar{\forall})$ ensure that $z$ is not free in $\gamma$ and by the usual restriction $z$ is not among the free variables of $\Gamma$, which appear free in $|\Gamma|_{\boldsymbol{v}}^{\gamma}$ as well. Since $z$ does not appear in the list of challenge variables for $|\forall z A|$, essential is also that $z$ cannot be free in the witnesses $\boldsymbol{a}$ from $|A|_{\boldsymbol{x}}^{\boldsymbol{a}}$.

\section{Future Work: Extension and Automation}

We can also consider other modalities, e.g., Howard $\left(!_{h}\right)$ and Diller-Nahm $\left(!_{d}\right)$, together with their duals $?_{h}$ and $?_{d}$. We assume a general ordering on all four modalities as $k>h>d>g$ and add the following weakening rules w.r.t. this partial order:

$$
\frac{\Gamma, ?_{i} A}{\Gamma, ?_{j} A} \quad \frac{\Gamma, !_{j} A}{\Gamma, !_{i} A} \quad(j>i \text { and } i, j \in\{k, h, d, g\})
$$

meaning that anytime we can choose to "forget" some information we had. The interpretation of the new exponentials should be, following [13], as follows:

$$
\begin{aligned}
\left|!_{d} A\right|_{\boldsymbol{f}}^{\boldsymbol{x}}: \equiv ! \forall \boldsymbol{y} \in \boldsymbol{f} \boldsymbol{x}|A|_{\boldsymbol{y}}^{\boldsymbol{x}} & \left|?_{d} A\right|_{\boldsymbol{y}}^{\boldsymbol{f}}: \equiv ? \exists \boldsymbol{x} \in \boldsymbol{f} \boldsymbol{y}|A|_{\boldsymbol{y}}^{\boldsymbol{x}} \\
\left|!_{h} A\right|_{\boldsymbol{f}}^{\boldsymbol{x}}: \equiv ! \forall \boldsymbol{y} \leq^{*} \boldsymbol{f} \boldsymbol{x}|A|_{\boldsymbol{y}}^{\boldsymbol{x}} & \left|?_{h} A\right|_{\boldsymbol{y}}^{\boldsymbol{f}}: \equiv ? \exists \boldsymbol{x} \leq^{*} \boldsymbol{f} \boldsymbol{y}|A|_{\boldsymbol{y}}^{\boldsymbol{x}}
\end{aligned}
$$

In some cases, when decidability of formulas is an issue, we might need to use the Diller-Nahm interpretation instead of Dialectica. Consider the following example (also (7) could serve as an example, if we assume that $f(m)=f(m+1)$ is undecidable)

$$
\forall f^{\mathbb{N} \rightarrow \mathbb{R}}(\forall m(f(m)<\mathbb{R} f(m+1)) \rightarrow \forall n(f(n)<\mathbb{R} f(n+2))) .
$$


Note that $<_{\mathbb{R}}$ is an undecidable relation. The best we can do is to collect a finite set of witnesses for $m$ (as functions of $n$ ). Like in Example 4 above, also here are we not interested in the redundant information hidden within $f(m)<\mathbb{R} f(m+1)$. For the sake of program-extraction, formula (10) is thus better labelled as

$$
\forall f^{\mathbb{N} \rightarrow \mathbb{R}}\left(!_{d} \forall m ?_{k}(f(m)<\mathbb{R} f(m+1)) \multimap \forall n ?_{k}(f(n)<\mathbb{R} f(n+2))\right) .
$$

We can produce a finite collection of witnesses for $m$ as $\Phi(f, n):=\{n, n+1\}$ so that

$$
\forall f^{\mathbb{N} \rightarrow \mathbb{R}}, n(\forall m \in \Phi(f, n)(f(m)<\mathbb{R} f(m+1)) \rightarrow(f(n)<\mathbb{R} f(n+2))) .
$$

Note that the same effect can be achieved via a light Diller-Nahm interpretation, using an $\bar{\exists}$ for the existential quantifier hidden within $<_{\mathbb{R}}$, rather than the Kreisel whynot ${ }_{k}$.

\subsection{Automated Decoration of Modalities}

Given a proof of a mathematical theorem, once the desired information (i.e., quantified variables to be realized) is selected, we can automatically view the intuitionistic proof as a hybrid linear logic proof, with the modalities decorated in such way that the proof analysis will give us the information requested. For instance, in a theorem of the form

$$
\forall x A \rightarrow \forall y \exists z B
$$

it could be that we are interested only in the negative universal information $x$, and not in the positive existential information $z$. Hence we rather present (11) as a specification in multi-modal linear logic decorated like

$$
!_{g} \forall x A \multimap \forall y ?_{k} \exists z B \quad \text { or } \quad !_{g} \forall x A \multimap \forall y \bar{\exists} z B .
$$

An automated tool can try to figure out if such a labelling of the given proof is possible. If it is, the hybrid interpretation will then return the realizer $t$ and a linear logic proof of

$$
\forall y(! A[t y / x] \multimap ? \exists z B) \quad \text { or } \quad \forall y(! A[t y / x] \multimap \exists z B)
$$

which can finally be translated back to an intuitionistic proof of $\forall y(A[t y / x] \rightarrow \exists z B)$.

The input to such a "decorating algorithm" is the intuitionistic proof of an intuitionistic formula $A$ and $A^{\star}$, a (light) hybrid decoration of the linear logic translation of $A$. We would like to transform the proof of $A$ into a (light) hybrid linear proof of $A^{\star}$. For this we should establish how the rules of intuitionistic logic could be translated to proofs in hybrid linear logic. In general, an intuitionistic proof of $B$ from uncancelled assumptions $A_{0}, \ldots, A_{n}$ gets canonically translated to a linear proof of $B$ from $!_{0} A_{0}, \ldots, !_{n} A_{n}$, where $!_{i}$ is one of the possible modalities, hence a proof of the sequent ${ }_{0} A_{0}^{\perp}, \ldots, ?_{n} A_{n}^{\perp}, B$. Whenever a linear cut rule is to be applied, one has to make sure that the exponential flavours in the cut formula from the left sub-tree are isomorphically corresponding to the exponential flavours in its linear negation from the right sub-tree. Moreover, this correspondence has to be coordinated recursively down into the sub-trees. It is nevertheless not enough to simply ensure that the exponential flavours propagate soundly from conclusion to axioms and assumptions. One has to also verify the various restrictions: those involved by contraction rules like $\mathrm{con}_{g}$ and, if the nc-quantifiers are used as well, those involved by the $(\bar{\forall})$ introduction rule.

Sometimes, hybrid decorated specifications $A^{\star}$ simply fail to have a hybrid proof. 


\section{Conclusion}

Hybrid interpretations successfully combine peculiar features of different functional interpretations. A few restrictions need to be satisfied when mixing the corresponding distinct modalities. The possibility of "colouring" the exponentials in a linear proof translation of the given specification with the desired flavours can be investigated by an algorithm. The non-computational quantifiers smoothly add to the picture in a way that uniformly explicates the structure of both light Dialectica and light modified realizability. Illustrative applications of the hybrid interpretations were here presented.

Example 3 brings an important optimisation of the usual treatment of induction by Dialectica. Full extensionality and the Markov principle are simultaneously treated under certain restrictions. Although not previously noticed, similar effects could be achieved via the nc quantifiers, but using the hybrid modalities appears to be smoother.

The user of hybrid interpretations thus has a large choice of techniques. The hunt for new applications has now just opened and the reader is warmly invited!

\section{References}

1. Oliva, P.: Unifying functional interpretations. Notre Dame Journal of Formal Logic 47(2), 263-290 (2006)

2. Kreisel, G.: Interpretation of analysis by means of constructive functionals of finite types. In: Heyting, A. (ed.) Constructivity in Mathematics, pp. 101-128. North Holland, Amsterdam (1959)

3. Gödel, K.: Über eine bisher noch nicht benützte Erweiterung des finiten Standpunktes. Dialectica 12, 280-287 (1958)

4. Girard, J.Y.: Linear logic. Theoretical Computer Science 50(1), 1-102 (1987)

5. Oliva, P.: Modified realizability interpretation of classical linear logic. In: Proc. of the 22nd Annual IEEE Symposium on Logic in Computer Science (LICS 2007). IEEE Press (2007)

6. Oliva, P.: An analysis of Gödel's Dialectica interpretation via linear logic. Dialectica (2008), http: //www.dcs.qmul.ac.uk/ pbo

7. Howard, W.A.: Hereditarily majorizable functionals of finite type. In: Troelstra, A.S. (ed.) WG 1988. Lecture Notes in Mathematics, vol. 344, pp. 454-461. Springer, Berlin (1973)

8. Danos, V., Joinet, J.B., Schellinx, H.: The structure of exponentials: Uncovering the dynamics of linear logic proofs. In: Mundici, D., Gottlob, G., Leitsch, A. (eds.) KGC 1993. LNCS, vol. 713, pp. 159-171. Springer, Heidelberg (1993)

9. Girard, J.Y.: Towards a geometry of interaction. Contemporary Mathematics 92 (1989)

10. Kohlenbach, U., Oliva, P.: Proof mining: a systematic way of analysing proofs in mathematics. Proceedings of the Steklov Institute of Mathematics 242, 136-164 (2003)

11. Berger, U.: Uniform Heyting Arithmetic. Annals Pure Applied Logic 133, 125-148 (2005)

12. Hernest, M.D.: Optimized programs from (non-constructive) proofs by the light (monotone) Dialectica interpretation. PhD Thesis, École Polytechnique and Universität München (2006), http://www.brics.dk/ danher/teza/

13. Oliva, P.: Computational interpretations of classical linear logic. In: Leivant, D., de Queiroz, R. (eds.) WoLLIC 2007. LNCS, vol. 4576, pp. 285-296. Springer, Heidelberg (2007)

14. Berger, U., Schwichtenberg, H., Buchholz, W.: Refined program extraction from classical proofs. Annals of Pure and Applied Logic 114, 3-25 (2002)

15. Hernest, M.D.: Light Dialectica program extraction from a classical Fibonacci proof. Electronic Notes in Theoretical Computer Science 171(3), 43-53 (2007) 